\title{
Kombinierte Fertigungslinien als neue Strategie in flexiblen Fertigungssystemen
}

\author{
DiPL.-Ing. AyMAN Al KhATEeB \\ FACHGEBIET FABRIKBETRIEB \\ TECHNISCHE UNIVERSITÄT ILMENAU, DEUTSCHLAND
}

\section{Zusammenfassung}

In Fertigung und Montage dienen Puffer einerseits der Entkopplung von Prozessschritten, andererseits der Vorbeugung negativer Wirkungen von Störungen. Ziel ist es stets, einen effizienten Ablauf mit hohem Durchsatz bei guter Wirtschaftlichkeit der Anlagen zu erreichen.

In Fabriken, die mit identischen Fertigungslinien zur Herstellung gleicher Produkte oder Werkstücke ausgerüstet werden, besteht die Möglichkeit, das Prinzip der vernetzten Pufferung anzuwenden. Die Kombination von zwei identischen Fertigungslinien mittels gemeinsam genutzter Puffer steht im Mittelpunkt der Auslegung des neuen Modells. Zunächst führt diese Kombination zur Senkung der erforderlichen Pufferkapazitäten. Die Folge davon sind die Reduzierung der Puffer um die Hälfte der ursprünglichen Kapazitäten, Verkleinerung des Flächenbedarfs, Verminderung von Störungswirkungen, eine wirtschaftlich mögliche Erhöhung des Durchsatzes und schließlich dadurch eine Verringerung der Investitionskosten.

\begin{abstract}
In manufacturing and assembly the buffers are used on the one hand for the decoupling of process steps, on the other hand to avoid the negative effects of failures. The aim is always to reach an efficient work of the equipment with high throughput by good economy.

Factories with identical manufacturing lines producing equal products or workpieces offer the possibility of applying the principle of the buffer network. The configuration of the new model is focussed on the combination of two common identical manufacturing lines which are combined by joint buffers. Initially, this combination entails a reduction of required buffer size which finally results in a reduction of original buffer capacities by half, reduction of space required, reduced effects of failures, economically practicable increase in throughput and thus a reduction in capital expenditure.
\end{abstract}

\section{Einleitung}

Ausgehend von den Möglichkeiten der Verkettung wird auf die Anordnung von Puffern im Anlagenlayout eingegangen. Es schließt sich die Entwicklung einer kombinierten Struktur an. Zur Untersuchung dieser Struktur werden methodisch sowohl analytische Vorgehensweisen als auch die Simulation angewendet. Ein Vergleich der Strukturen führt zu eindeutigen Ergebnissen im Hinblick auf Flächenbedarf, Durchsatz und Störungsfolgen.

Flexible Fertigungssysteme können durch Weiterentwicklung des Produktionsprinzips verbessert werden (siehe z.B. [Tuff.1988]). Um die Zwischenlagerzeiten zwischen Bearbeitungsstationen zu reduzieren, hatte [Adam.1993] die Tendenz zu einer vernetzten Fertigung, sodass die Bewegungen von Werkstücken innerhalb der Gruppenfertigung flexibel ausgeführt werden. In diesem Beitrag wird eine neue Strategie zur Optimierung der identischen Fertigungslinien bei verschiedenen Fertigungsarten entwickelt.

Hauptkomponenten der flexiblen Fertigungslinie sind Bearbeitungsstationen, die durch Zwischenpuffer miteinander verbunden werden (siehe [Renn.1986]). Jede Bearbeitungsstation besteht aus einer oder mehreren Bearbeitungseinheiten (Maschinen, Menschen). Zwischenspeicher dienen als ein Puffer zur Aufnahme eines oder mehrere Werkstücke bzw. Werkstückträger, rein zur Zeit-Überbrückung. Zur Erstellung einer Fertigungslinie werden meist eine oder mehrere von den folgenden konventionellen Strukturen verwendet (siehe [Kuhn.1998], [Salv.2001]):

Starre Verkettung von Bearbeitungsstationen (Abbildung 1.a):

Es geht um eine feste Reihenfolge von Bearbeitungsstationen, in denen die Bearbeitung der Werkstücke erfolgt. Die Bewegung eines Werkstücks von einer Station zur nächsten wird vom Zustand der jeweiligen Folgestation beschränkt; d.h. von deren Zustand (noch mit der Bearbeitung eines anderen Werkstücks beschäftigt bzw. frei oder gestört). 
Lose bzw. elastische Verkettung von Bearbeitungsstationen (Abbildung 1.b):

Die Stationen werden durch Puffer verbunden. Das Hauptziel dieser Puffer besteht darin, die Wartezeiten einer Station auf Werkstücke bzw. die Blockierung eines Werkstücks in der vorangehenden Station möglichst zu vermeiden. Es werden unterschiedliche Bearbeitungszeiten zwischen den Stationen durch Puffer ausgeglichen.

Verzweigte (nicht lineare) Verkettung von Bearbeitungsstationen (Abbildung 1.c):

In dieser Struktur kann ein Puffer von mehreren Stationen genutzt werden, das heißt, er wird als Zugangspuffer für viele Stationen vorgesehen. Außerdem kann mehrstufiges Puffern von einer oder mehreren Stationen benutzt werden. Die Abbildung 1.c zeigt eine Möglichkeit der Verwendung eines Ausweichpuffers (siehe [Furm.1992]) von linearen und nicht linearen Fertigungslinien.

(a)-

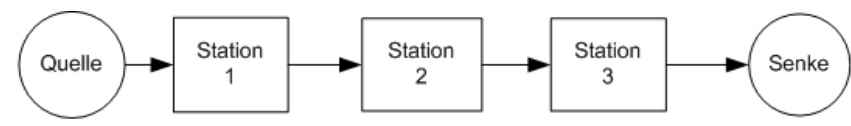

(b)-

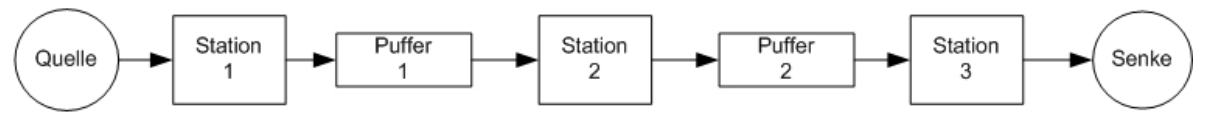

(c)-

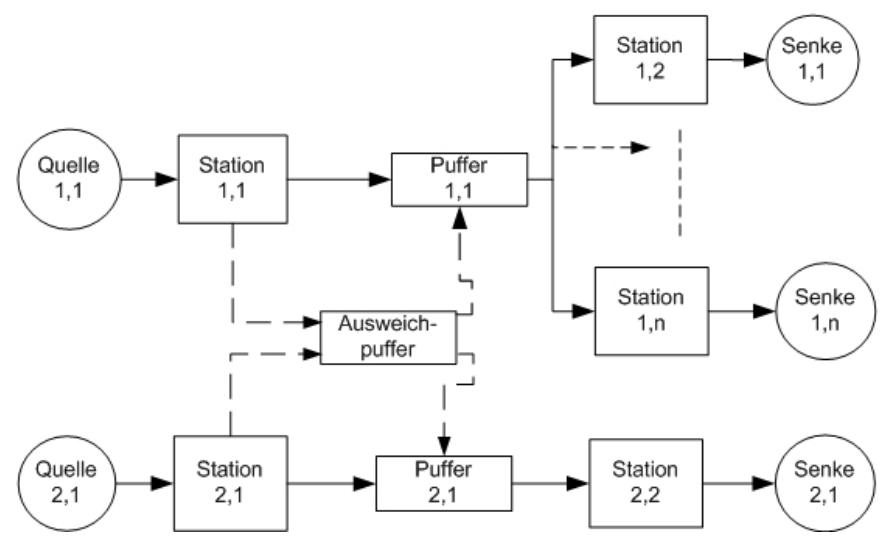

Abbildung 1: Konventionelle Strukturen der Verkettung von Bearbeitungsstationen in den Fertigungslinien

\section{Kombinierte Fertigungslinien durch Puffer}

In der Fertigungslinie dienen die Puffer zum Ausgleich verschiedener Bearbeitungszeiten an aufeinander folgenden Stationen. Damit mag ein Puffer zwischen jeweils zwei Stationen liegen (Abbildung 1.b), sodass sich die Komponentenanzahl der Fertigungslinien verdoppelt und schließlich die Investitionskosten erhöhen. Unter Betrachtung mehrerer Produktionslinien zur Herstellung gleicher Produkte in einer Fabrik besteht der Bedarf, diese Linien zu optimieren und die Kosten zu reduzieren. Dafür werden jeweils zwei Linien miteinander durch Puffer verbunden. Es werden die nachstehend aufgeführten Annahmen getroffen, um das neue Modell zu erstellen:

- Die beiden Fertigungslinien sind identisch, sodass sie dieselben Komponenten und Nutzungsbedingungen haben.

- Die beiden Linien führen gleichartige Aufträge aus, sodass gleiche Produkte hergestellt werden.

Um das neue Modell zu bauen, werden jeweils zwei nebeneinander liegende Stationen der beiden Linien (symmetrische Anordnung) mit einem gemeinsamen Abgangspuffer verbunden. Dafür werden die in einer von den beiden Linien vorhandenen Puffer als gemeinsame Abgangspuffer verwendet (siehe Abbildung 2). Es ist außerdem möglich, die Puffergrößen zu verändern.

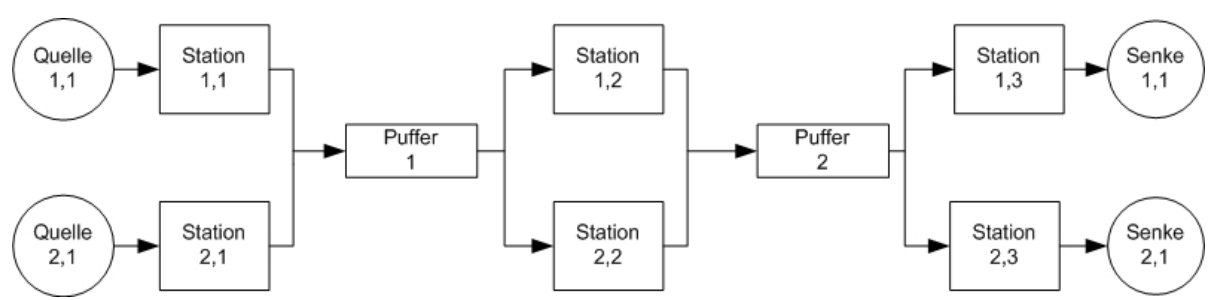

Abbildung 2: Die neue kombinierte Struktur 


\section{Reduzierung der geplanten Pufferkapazitäten um die Hälfte}

Das Ziel, die Anzahl der Puffer auf die Hälfte zu reduzieren, bedeutet nicht eine notwendige Erhöhung ihrer Pufferkapazitäten, um den zuvor erreichten Durchsatz beizubehalten.

Solange die Verteilung der Bearbeitungszeiten der beiden parallelen Bearbeitungsstationen nicht konstant sind (z.B. können sie generell oder erlangverteilt sein), treten die von den Stationen ankommenden Werkstücke meist nicht gleichzeitig in den gemeinsamen Puffer ein. Und analog verlassen sie ihn nicht gleichzeitig. Die Bewegungen der Werkstücke aus und in den gemeinsamen Puffer hängen von der Pufferkapazitäten und der Verteilung der Bearbeitungszeiten der mit demselben Puffer verbundenen Stationen ab. Die Berechnung der Puffergrößen wird unter Benutzung der bedientheoretischen ${ }^{1}$ Methode und Simulation ausgeführt. Mit Unterstützung der analytischen Methode können einige Materialflusssysteme untersucht werden, um die mittleren Puffergrößen nach hinreichend langer Zeitdauer zu berechnen. Damit sind für einfache Systeme gute Abschätzungen möglich, während die Simulation komplexere reale Systeme nachbilden und die optimalen Pufferkapazitäten in befristeter gewünschter Zeit ermitteln kann. Natürlich muss das Simulationsmodell das reale System hinreichend genau abbilden, um aus den Simulationsexperimenten Rückschlüsse auf dieses ziehen zu dürfen (vgl. [VDI 3633]).

\subsection{Verwendung der bedientheoretischen Methode}

In der Literatur findet man sowohl im Fertigungsbereich und in der Logistik wie z.B. [Furm.1992], [Liebe.2006] und [Gers.2003] als auch in der Informatik wie z.B. [Bolc.2006] mehrere Materialfluss- und Bediensysteme, die unter Verwendung der bedientheoretischen Methode analysiert werden. Es wird angenommen, dass zwei identische Fertigungslinien, die in der Abbildung 3 dargestellt werden, miteinander durch einen Puffer kombiniert werden. Man geht davon aus, dass die Verteilung von Bearbeitungszeiten der jeweiligen Bearbeitungsstation generell ist. Mittelwerte, Varianzen und Variationskoeffizienten sind lediglich die bekannten Parameter dieser Verteilung.

(a)
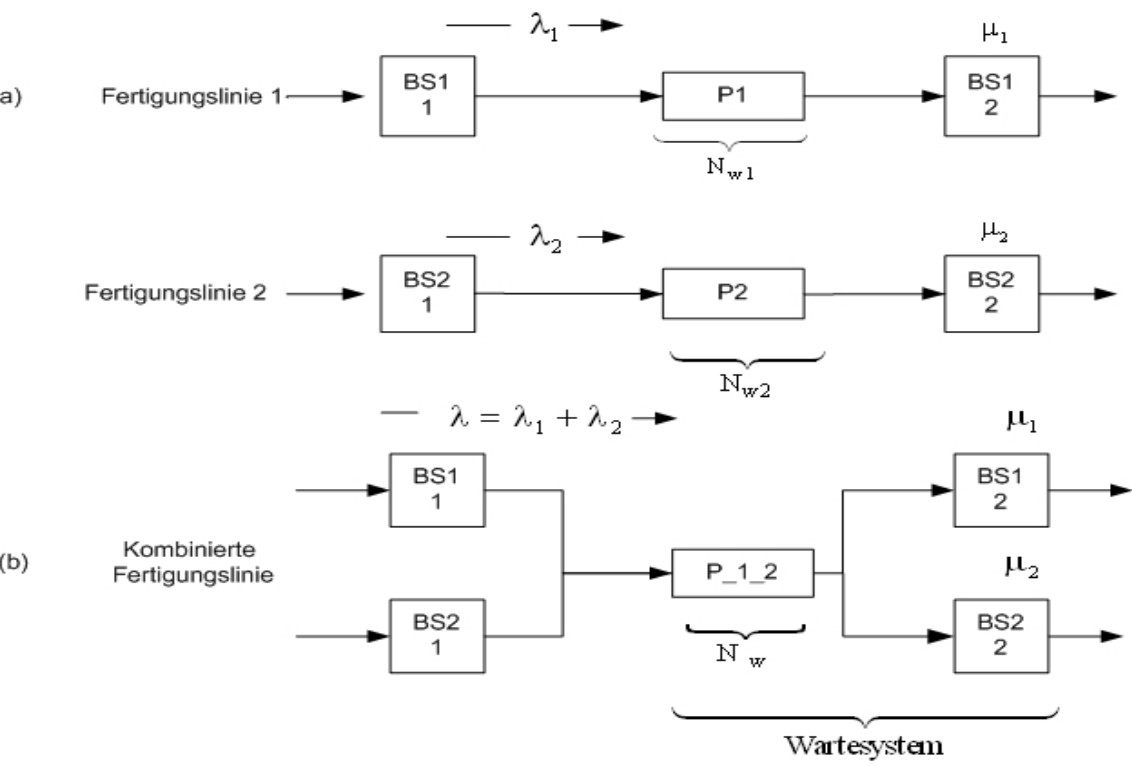

Abbildung 3: Zwei identische Fertigungslinien und ihre kombinierte Struktur

Trotz der praktischen Bedeutung der Verwendung von G/G/1- und G/G/m-Modellen in den Materialfluss-systemen werden noch keine bestimmten Formeln zur Berechnung ihre Parameter angegeben. Zur Untersuchung dieses Modells werden die folgenden Annahmen getroffen:

- $\quad \lambda_{1}=\lambda_{2}$ : Die Ankunftsrate der Werkstücke im Puffer (P1, P2) [Werkstück/Zeiteinheit]

- $\lambda=\lambda_{1}+\lambda_{2}$ : Die Ankunftsrate der Werkstücke im Puffer (P_1_2)

- $\mu_{1}=\mu_{2}=\mu$ : Die Bedienrate der Werkstücke in den Bearbeitungsstationen (BS12, Bs22) [Werkstück/Zeiteinheit]

- $\quad \mathrm{N}_{\mathrm{w} 1}, \mathrm{~N}_{\mathrm{w} 2:}$ Der Mittelwert der Größen der Puffer (P1, P2), wenn der Warteprozess stationär geworden ist (vergleich [Arno.2005])

- $\mathrm{N}_{\mathrm{w}}$ : Der Mittelwert der Größe des Puffers (P_1_2)

- Die Puffergrößen sind unbeschränkt; d.h. es gibt immer noch Plätze für neue Werkstücke.

\footnotetext{
${ }^{1}$ Basiert auf der Warteschlangentheorie oder sog. Bedienungstheorie.
} 
- Jede Fertigungslinie in der Abbildung 3 gilt als G/G/1-Modell. Analog gilt die kombinierte Fertigungslinie als $\mathrm{G} / \mathrm{G} / \mathrm{m}-$ Modell

- $\quad \mathrm{m}=2$ : Die Anzahl der Bearbeitungsstationen, die nach dem Puffer (P_1_2) liegen

- $\quad \mathrm{v}_{\mathrm{a}}=\frac{\sqrt{\operatorname{Var}\left(\mathrm{t}_{\mathrm{a}}\right)}}{\mathrm{E}\left(\mathrm{t}_{\mathrm{a}}\right)}\left(\mathrm{vgl}\right.$. [Arno.2005]): Variationskoeffizient der Ankunftsprozesse; $\mathrm{E}\left(\mathrm{t}_{\mathrm{a}}\right)$ : Mittelwert der Bearbeitungszeit von Bearbeitungsstationen, die vor dem Puffer liegen, $\operatorname{Var}\left(\mathrm{t}_{\mathrm{a}}\right)$ : Varianz des Mittelwerts

- $\mathrm{v}_{\mathrm{b}}=\frac{\sqrt{\operatorname{Var}\left(\mathrm{t}_{\mathrm{b}}\right)}}{\mathrm{E}\left(\mathrm{t}_{\mathrm{b}}\right)}$ : Variationskoeffizient der Bearbeitungsprozesse; $\mathrm{E}\left(\mathrm{t}_{\mathrm{b}}\right)$ : Mittelwert der Bearbeitungszeit von Stationen, die nach dem Puffer liegen, $\operatorname{Var}\left(\mathrm{t}_{\mathrm{b}}\right)$ : Varianz des Mittelwerts.

Ist die Auslastung $\left(\rho_{1}=\frac{\lambda_{1}}{\mu_{1}}<1\right.$ und $\rho_{\rho_{2}}=\frac{\lambda_{2}}{\mu_{2}}<1$ ) für das G/G/1-Modell in der Abbildung 3.a richtig, so ist $\rho=\frac{\lambda_{1}+\lambda_{2}}{\mu_{1}+\mu_{2}}=\frac{\lambda}{2 \mu}<1$ im G/G/m-Modell in der Abbildung 3.b auch richtig. Wenn der Warteprozess stationär (eingeschwungener Zustand) und die Anzahl der Werkstücke (K) im Wartesystem ( $\mathrm{k} \geq \mathrm{m}$ ) sind, wird der Auslastungsgrad nach [Arno.2005] berechnet: $\rho=\frac{\lambda}{\mathrm{m} \mu}$

Viele Approximationen werden von [Bolc.2006] erwähnt, um die Mittlere Wartezeit eines Werkstücks ( $\overline{\mathrm{W}}$ ) zu bearbeiten z.B.:

Approximation von Allen-Cunneen (A-C) Formel:

$\overline{\mathrm{W}} \approx \frac{\mathrm{P}_{\mathrm{m}} / \mu}{1-\rho} \frac{\mathrm{v}_{\mathrm{a}}^{2}+\mathrm{v}_{\mathrm{b}}^{2}}{2 \mathrm{~m}} ;\left(\mathrm{P}_{\mathrm{m}}\right)$ Die Wahrscheinlichkeit, dass ein Werkstück nicht sofort bearbeitet werden kann $(\mathrm{k}>\mathrm{m})$

Approximation von Krämer-Langenbach-Belz:

$$
\begin{aligned}
& \overline{\mathrm{W}} \approx \frac{\mathrm{P}_{\mathrm{m}} / \mu}{1-\rho} \frac{\mathrm{v}_{\mathrm{a}}^{2}+\mathrm{v}_{\mathrm{b}}^{2}}{2 \mathrm{~m}} \mathrm{G}_{\mathrm{KLB}} ; \\
& \mathrm{G}_{\mathrm{KLB}}= \begin{cases}\exp \left(-\frac{2}{3} \frac{1-\rho}{\left.\mathrm{P}_{\mathrm{m}} \frac{\left(1-\mathrm{v}_{\mathrm{a}}^{2}\right)^{2}}{\mathrm{v}_{\mathrm{a}}^{2}+\mathrm{v}_{\mathrm{b}}^{2}}\right),}\right. & 0 \leq \mathrm{v}_{\mathrm{a}} \leq 1, \\
\exp \left(-(1-\rho) \frac{\mathrm{v}_{\mathrm{a}}^{2}-1}{\mathrm{v}_{\mathrm{a}}^{2}+4 \mathrm{v}_{\mathrm{b}}^{2}}\right), & \mathrm{v}_{\mathrm{a}}>1\end{cases}
\end{aligned}
$$

Die Berechnung von $\mathrm{N}_{\mathrm{w}}$ erreicht man nach Little's Gesetz: $\mathrm{N}_{\mathrm{w}}=\lambda \overline{\mathrm{w}}$

Um die Ersparnis der Puffergröße in der kombinierten Fertigungslinie zu ermitteln, werden die Werte $\left(\mathrm{N}_{\mathrm{w}}\right)$ in den speziellen Fällen $(\mathrm{m}=1$ und $\mathrm{m}=2)$ berechnet und miteinander verglichen. Nach [Bolc.2006] ergibt sich das Diagramm 1. Dieses Diagramm zeigt, dass je größer der Wert (m) ist, desto größer ist die Ersparnis der Puffergröße, wenn der Auslastungsgrad $\rho$ konstant ist $(\rho=\rho 1=\rho 2)$. Der Mittelwert der Pufferkapazität in der kombinierten Fertigungslinie kann um mehr als die Hälfte reduziert werden.

\subsection{Verwendung der Simulation}

Mit der Verwendung der Simulation kann man reale Bedingungen nachbilden und ausreichende Detaillierung visualisieren. Man geht davon aus, dass die Pufferkapazitäten in der Abbildung 4 beschränkt sind. Sind die Bearbeitungszeiten von je zwei aufeinander folgenden Stationen nahezu gleich (gleicher Mittelwert und unterschiedliche Abweichung), so ermöglicht die kombinierte Struktur der Fertigungslinien eine signifikante Reduzierung der geplanten Pufferkapazitäten um die Hälfte.

Beispiel: Es wird angenommen, dass der Mittelwert der Bearbeitungszeiten der Stationen A, C, D, L, M, N, A1, C1, D1, L1, M1 und N1 (siehe Abbildung 4) 30 s erlangverteilt, die Standardabweichung $10 \mathrm{~s}$, die untere Schranke $20 \mathrm{~s}$ und die obere Schranke $40 \mathrm{~s}$ ist. Die geplanten Kapazitäten jeweils zweier symmetrischer Puffer (P2, P5 und P3, P6) in zwei konventionellen Fertigungslinien sind 10 Speicherplätze. Das heißt, die Summe beträgt 20 Speicherplätze je Fertigungslinie. Diese Pufferkapazitäten wurden nach den Diagrammen 2 und 3 so ausgewählt, dass ein möglichst großer Durchsatz unter der Berücksichtigung der Erhöhung der Pufferauslastung ist (vgl. Diagramm 4) und dadurch die reduzierten Pufferkosten erreichbar sind.

Beträgt die Kapazität der jeweiligen gemeinsamen Puffer (P 2_5, P 3 6) in der kombinierten Struktur 10 Speicherplätze, so wird ein fast gleicher Durchsatz im Vergleich mit der konventionellen Struktur erreicht. Die zu verbringenden Zeiten in den Puffern (Bearbeitungs-, Erhol-, oder Zykluszeit) wurden als vernachlässigbar angenommen. 


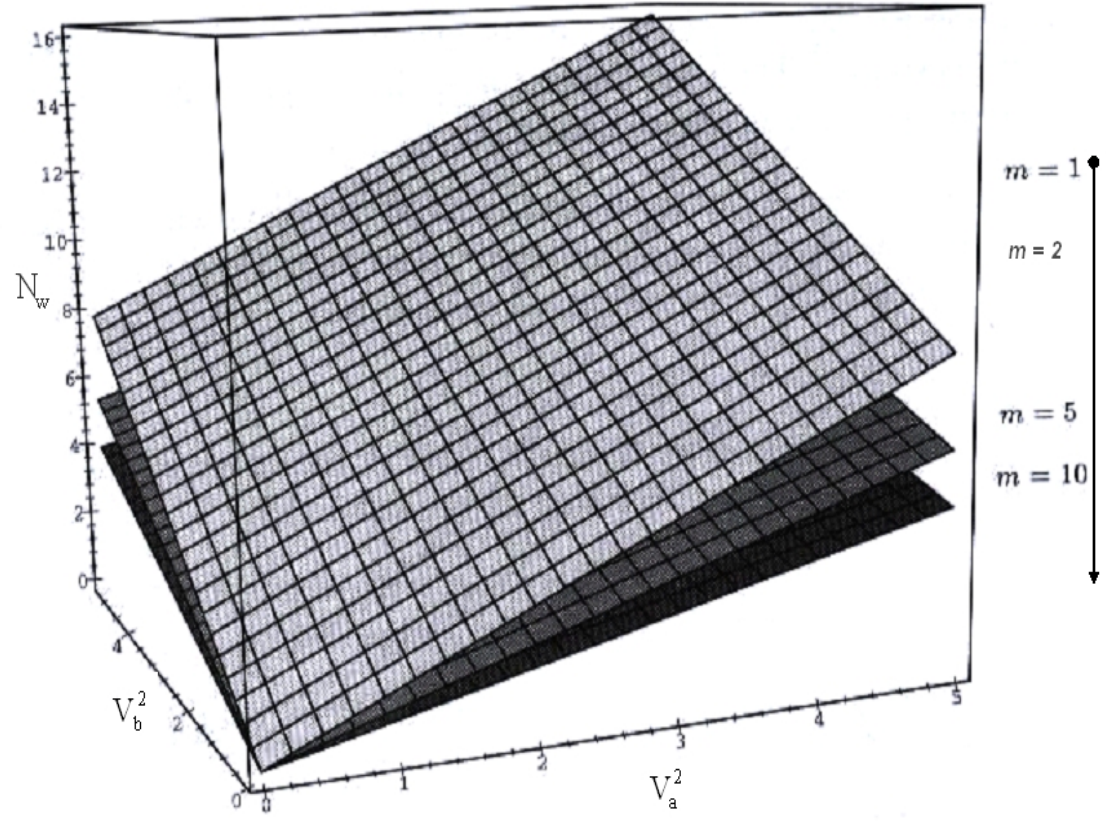

Diagramm 1: Mittelwert der Anzahl von Werkstücken $\left(\mathrm{N}_{\mathrm{w}}\right)$ für das $\mathrm{G} / \mathrm{G} / \mathrm{m}-\mathrm{Modell}(\mathrm{mit} \rho=0,8)$ nach [Bolc.2006]

Betrachtet man die Diagramme 2 und 3, erkennt man:

- Mit wachsenden Pufferkapazitäten in einer Fertigungslinie erhöht sich auch der Durchsatz.

- Es gibt jedoch eine optimale Pufferkapazität, sodass eine weitere Vergrößerung dieser Kapazität keine oder lediglich eine geringe, zu vernachlässigende Verbesserung des Durchsatzes ergibt.

- In der neuen Struktur sind die Pufferkapazitäten, um die Hälfte zu reduzieren.

Durch die Kombination ergibt sich eine höhere Flexibilität in der Versorgung von Folgeprozessen, die sich hier (Puffer P_2_5) in geringerer erforderlicher Pufferkapazität auswirkt.

Das Diagramm 4 zeigt eine Wahrscheinlichkeit der Häufigkeiten von Inhalten der letzten optimierten Puffer (P2, P5 und P_2 5) in den drei verschiedenen Fertigungslinien, die in der Abbildung 4 dargestellt wurden.

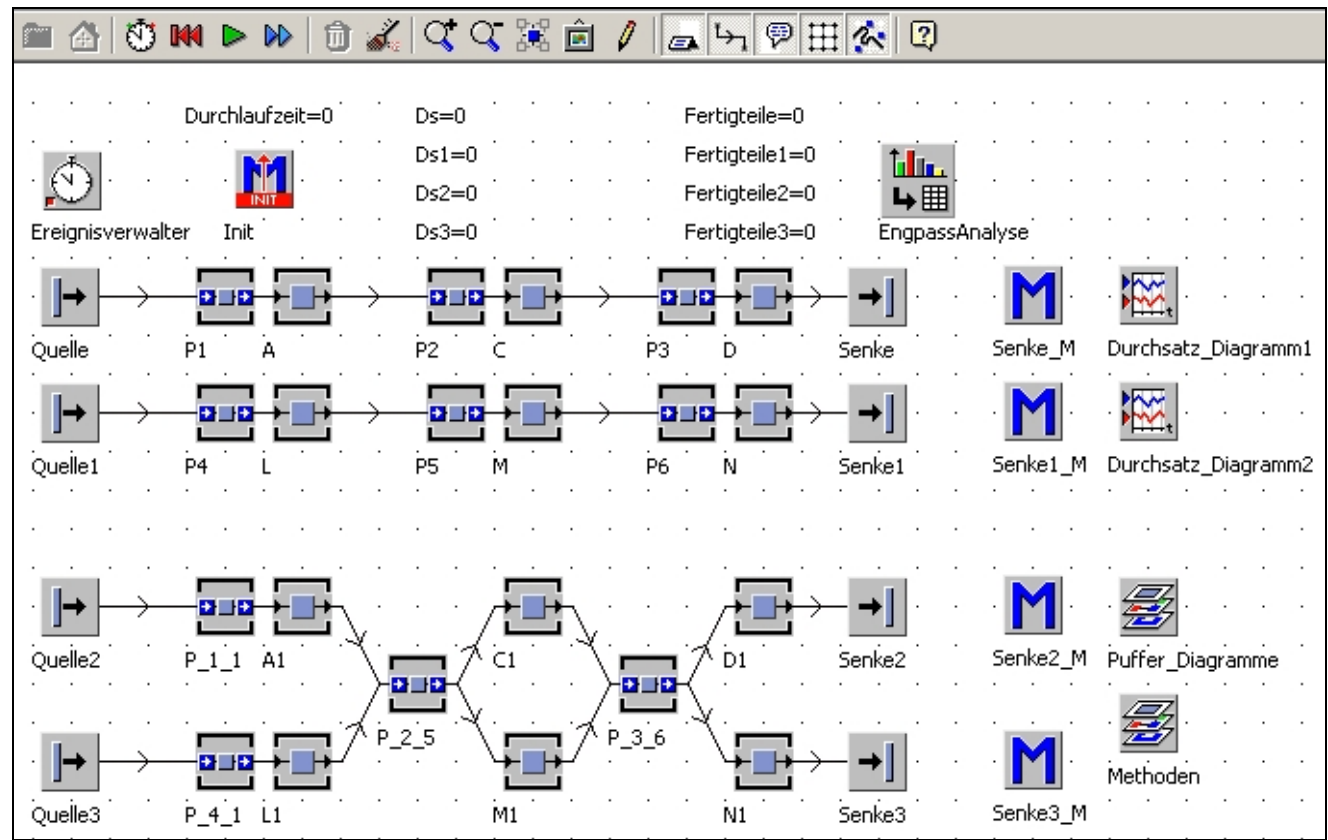

Abbildung 4: Zwei simulierten Fertigungslinien und ihre kombinierte Struktur. Ds und Ds1 sind Durchsätze [Stück/Stunde] am Ende der konventionellen Fertigungslinien, Ds2 und Ds3 sind Durchsätze [Stück/Stunde] am Ende der kombinierten Fertigungslinien. 


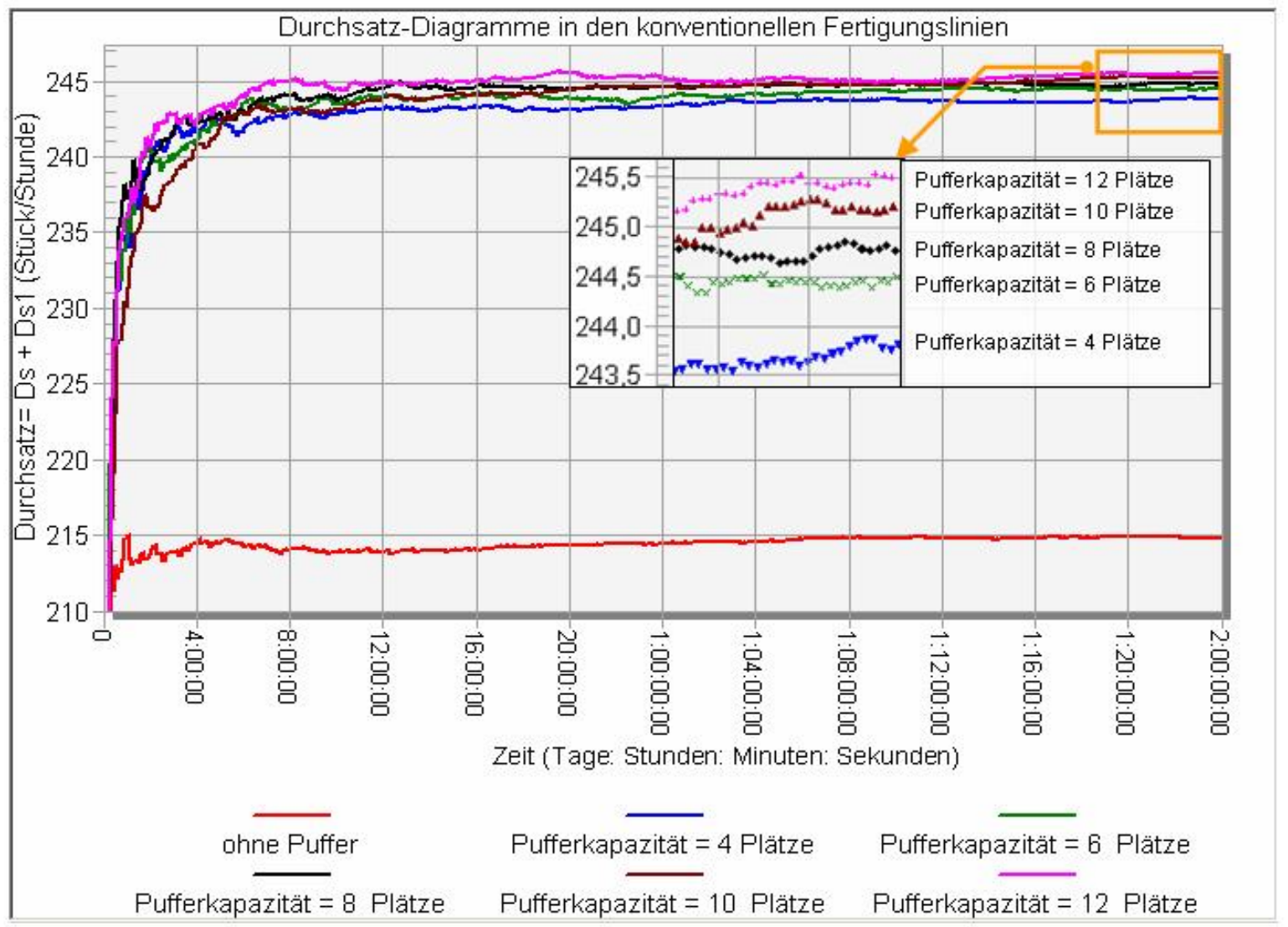

Diagramm 2: Die Beziehung zwischen Pufferkapazitäten und erreichbaren Durchsätzen in den konventionellen Fertigungslinien. Die Untersuchungsdauer beträgt 48 Stunden (2 Tage).

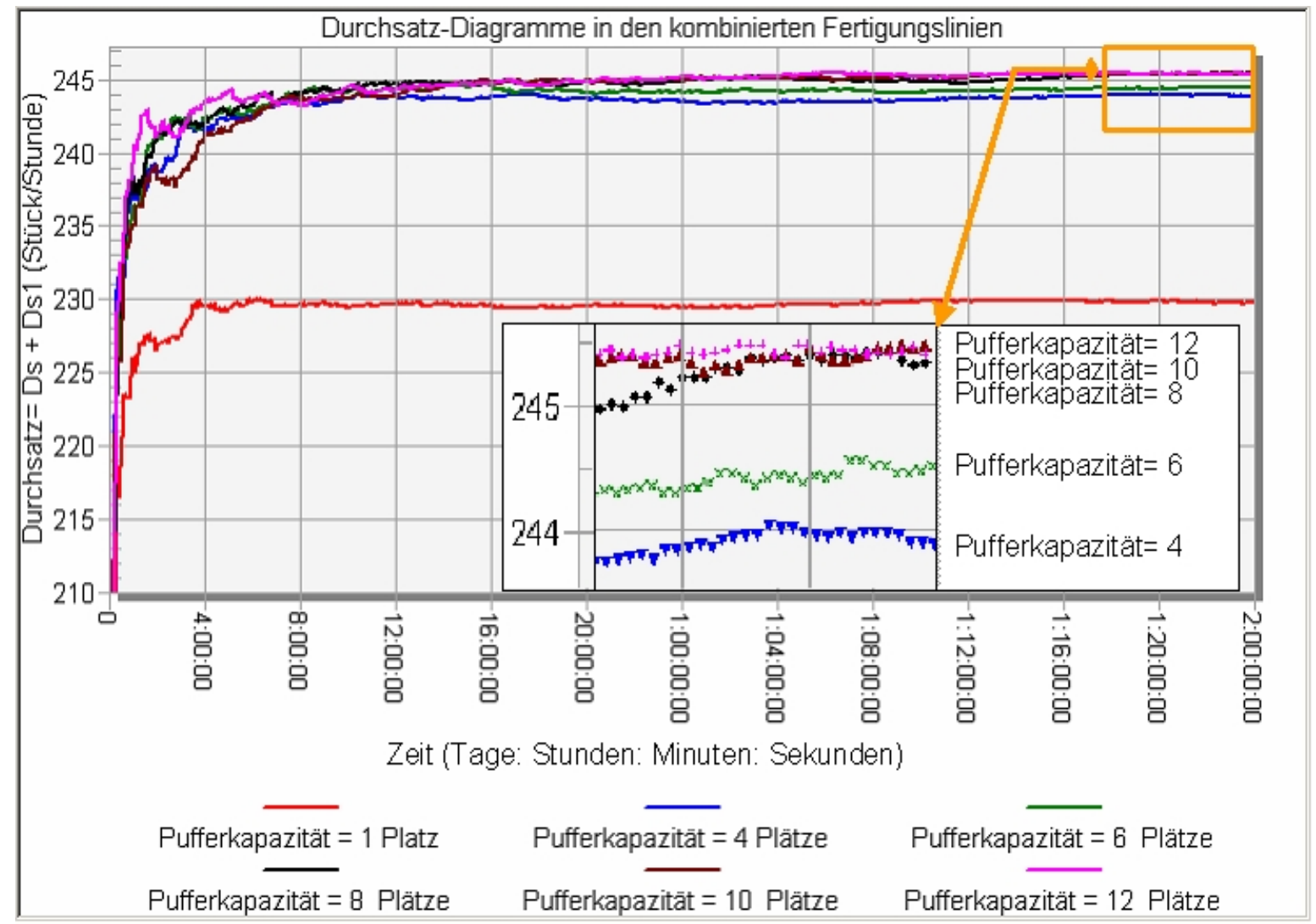

Diagramm 3: Die Beziehung zwischen Pufferkapazitäten und erreichbaren Durchsätzen in den kombinierten Fertigungslinien. Die Untersuchungsdauer beträgt 48 Stunden (2 Tage). 


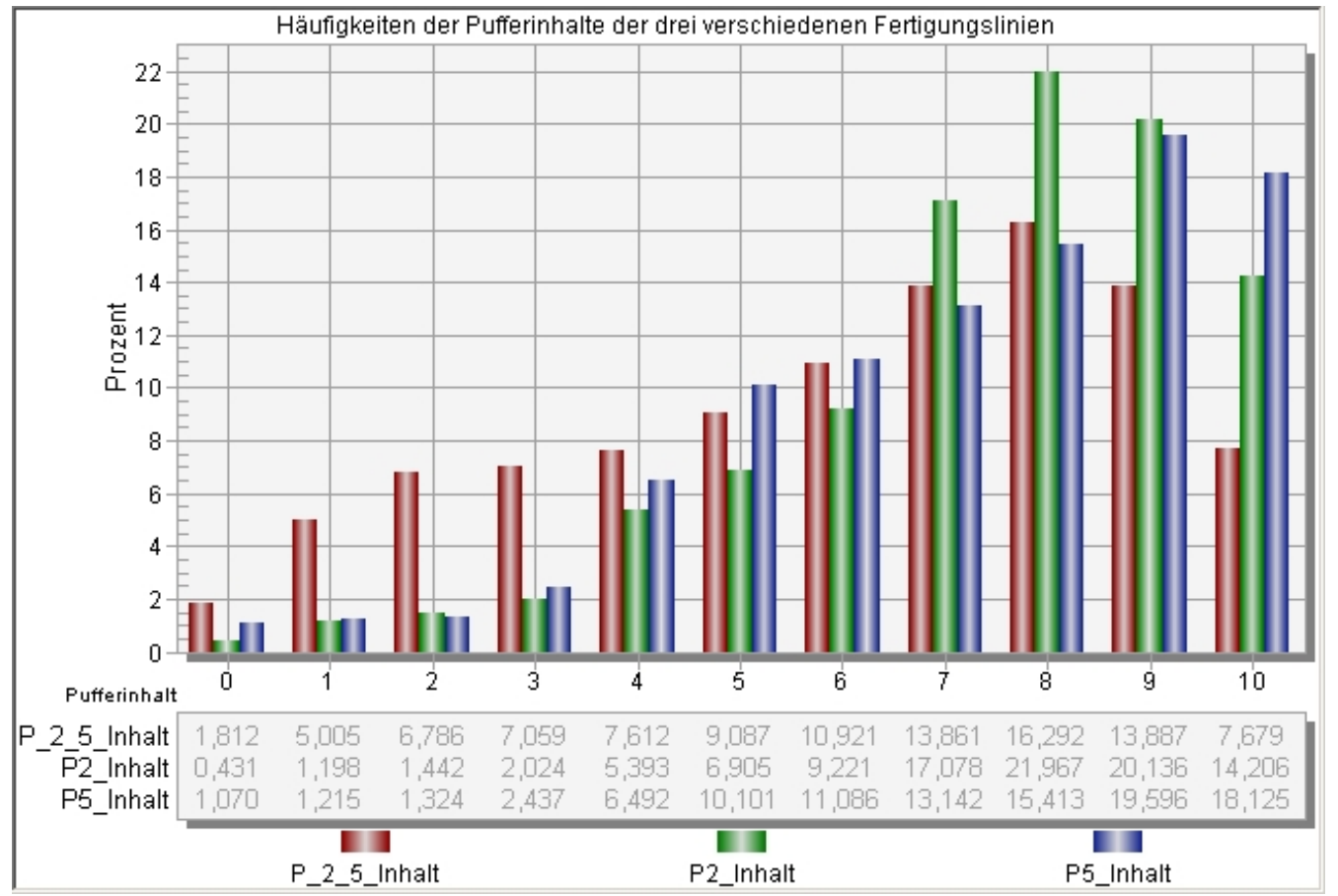

Diagramm 4: Wahrscheinlichkeiten der Häufigkeiten von Inhalten der Puffer P2, P5 und P_2_5 in den drei verschiedenen Fertigungslinien (Abbildung 4). Die Untersuchungsdauer beträgt 48 Stunden (2 Tage).

\section{Verkleinerung des Flächenbedarfs}

Die neue Struktur führt dazu, dass die Flächen der als nicht weiter erforderlich angesehenen Puffer und damit die zuvor zwischen der Fertigungslinie liegenden Flächen für diese Puffer gewonnen werden. Je größer die Differenz zwischen den Bearbeitungszeiten ist, desto größer ist die Pufferkapazität auszulegen. Diese Zunahme der Puffergröße ist erforderlich zur Erhöhung der Auslastung von Bearbeitungsstationen durch Verminderung der Blockierzeiten und Wartezeiten der Folgeprozesse und führt schließlich zur Erhöhung des Durchsatzes. Als wirtschaftliche Lösung in kombinierten Fertigungslinien stehen beispielsweise vertikale Puffer zur Verfügung.

\section{Weitere Erhöhung des Durchsatzes}

Da jeder Zugangspuffer in der kombinierten Fertigungslinie von zwei Stationen versorgt wird, reduziert sich die Wartezeit an den folgenden Stationen; deswegen entsteht eine Erhöhung des Durchsatzes. Dagegen schränkt sich diese Möglichkeit ein, wenn die Kapazität des gemeinsamen Puffers begrenzt ist, sodass sich die Blockierzeit wegen der Pufferüberlastung erhöht. Eine signifikante Steigerung des Durchsatzes kann aber in speziellen Fällen erreicht werden. Falls die Bearbeitungszeit einer Station (siehe Abbildung 1.b) größer als die Bearbeitungszeit der direkt vorgelagerten Station ist, aber kleiner als das Doppelte, sodass die Addition einer parallel gleichen Station, um den Durchsatz zu erhöhen, nicht wirtschaftlich ist, existiert in der neuen Struktur die Möglichkeit eine parallele Station zu beiden symmetrischen Stationen hinzuzufügen. Beispiel: Unter Annahme, dass der Mittelwert der Bearbeitungszeiten der Stationen A, L, A1 und L1 (siehe Abbildung 5) 20 s und der Stationen (C, M, C1, M1 und C2) 30 s erlangverteilt ist, kann sich der Durchsatz der gesamten kombinierten Fertigungslinie wirtschaftlich um bis zu 30\% vergrößern (siehe Diagramm 5).

\section{Verminderung der Störungswirkungen}

In dieser neuen Struktur werden je zwei parallele Stationen von einem Zugangspuffer mit Werkstücken versorgt und an einem Abgangspuffer liefern sie die bearbeiteten Werkstücke, wodurch keine direkten Verbindungen zwischen den Stationen entstehen (vgl. Abbildung 2). Wenn eine Störung an einer Station auftritt, wird ihre Wirkung auf die Fertigungslinie relativ gering sein, weil die Nachfolgestationen von dem gemeinsamen $\mathrm{Zu}-$ gangspuffer versorgt werden und gleichzeitig die Vorgängerstationen die Werkstücke im entsprechenden gemeinsamen Abgangspuffer abgeben. Außerdem kann die Kapazität des gemeinsamen Puffers (z.B. P_2_5 in der 


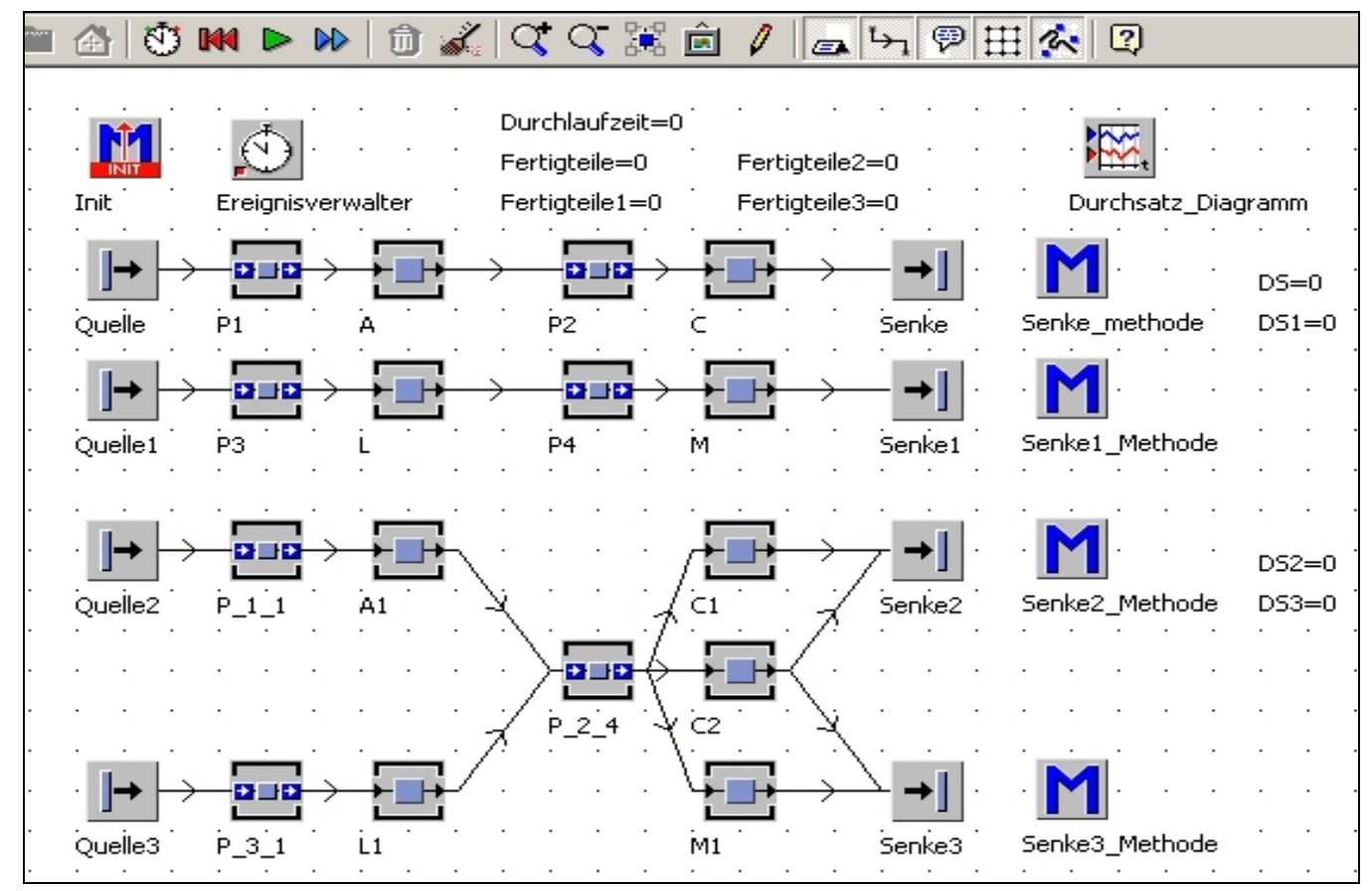

Abbildung 5: Eine Möglichkeit, eine weitere Station parallel zu beiden parallelen Stationen hinzuzufügen.

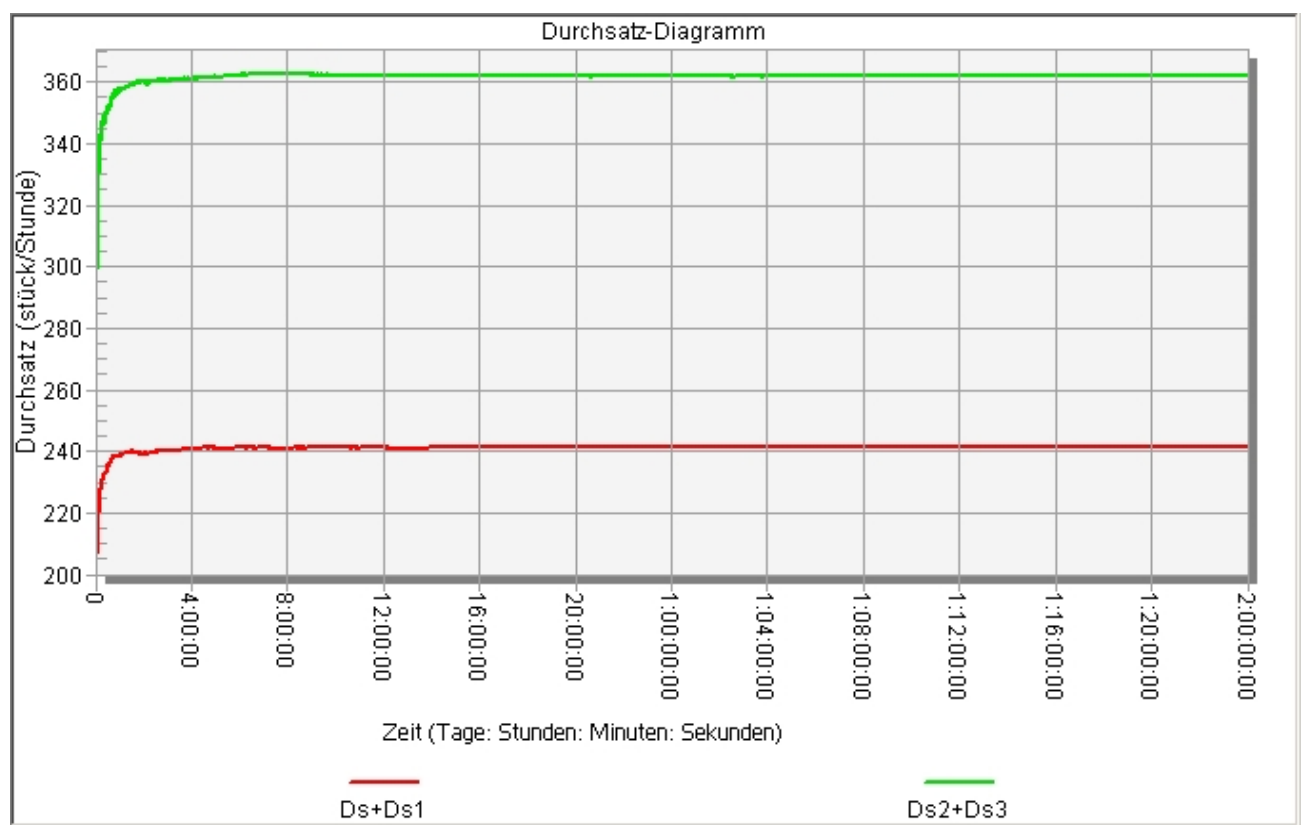

Diagramm 5: Möglichkeit, den Durchsatz wirtschaftlich zu erhöhen. Die Untersuchungsdauer beträgt 48 Stunden (2 Tage).

Abbildung 4) meist größer als die des jeweiligen Puffers (P2, P5) ausgelegt werden. Hauptsächlich beruhen die Störungswirkungen auf der Störungsdauer, den Kapazitäten der Zu- und Abgangspuffer der Stationen und auf der Ankunfts- und Bedienrate.

\section{Weitere Potentiale könnten im Folgenden liegen}

Die Entwicklung des neuen Modells ermöglicht zunächst die Optimierung der identischen Fertigungslinien, so dass die Leistung gesteigert wird und die Investitionskosten gleichzeitig verringert werden. Anschließend bietet sich die Möglichkeit, diese Struktur für nicht identische Fertigungslinien und Fließmontage einzusetzen, sodass Puffer mit gesteigerten Kapazitäten oder dezentrale Lager als gemeinsame Puffer benutzt werden. Hierbei kann jeder Platz des Puffers von jedem der verschiedenen Erzeugnisse belegt werden.

Der Fluss der Erzeugnisse bleibt unverändert wie er vor der Zusammenstellung der einzelnen Puffer gewesen wäre. Das heißt, dass im Einzelfall ein „,bestimmtes“ Erzeugnis auch nur zu einer (nach dem Arbeitsplan) bestimmten Station gefördert werden darf.

Als Vorteile sind auch hier zu sehen:

- Erhebliche Ersparnis des Pufferflächenbedarfs, da die Pufferauslastung steigt.

- Mögliche Verwendung von Lagertechnik. 


\section{Literatur}

[Adam.1993] Adam, Dietrich: Flexible Fertigungssysteme: Flexible Fertigungssysteme (FFS) im Spannungsfeld zwischen Rationalisierung, Flexibilisierung und veränderten Fertigungsstrukturen. Wiesbaden: Gabler, 1993. ISBN: 3-409-17914-3

[Arno.2005] Arnold, Dieter; Furmans, Kai: Materialfluss in Logistiksystemen (4. Auflage). Springer Berlin Heidelberg New York: 2005. ISBN: 3-540-22800-4

[Bolc.2006] Bolch, Gunter; Greiner, Stefan; de Meer, Hermann; S.Trivedi, Kishor: Queueing networks and Markov Chains: Modeling and performance evaluation with computer science applications. Hoboken, NJ : Wiley-Interscience, 2006. ISBN-13 978-0-471-56525-3, ISBN-10 0471-56525-3.

[Furm.1992] Furmans, Kai: Ein Beitrag zur Theoretischen Behandlung von Materialflusspuffern in Bediensystemnetzwerken. Karlsruhe: Inst. für Fördertechnik, 1992. ISSN 171-2772.

[Gers.2003] B.Gershwin, Stanley; Dallery, Yves; T. Papadopoulos, Chrissoleon; Smith., J.MacGregor: Analysis and modeling of manufacturing systems. Boston [u.a.] : Kluwer Acad. Publ., c 2003. ISBN: $1-4020-7303-8$.

[Kuhn.1998] Kuhn, Heinrich: Fließproduktionssysteme: Leistungsbewertung, Konfigurations- und Instandhaltungsplanung. Heidelberg : Physica-Verl., 1998. ISBN: 3-7908-1128-9.

[Libe.2006] Liberopoulos, George; Gershwin, Stanley B. ; Papadopoulos, Chrissoleon T. ; Smith, J. M. ; Tan, Baris: Stochastic modeling of manufacturing systems: advances in design, performance evaluation, and control issues. Berlin, Heidelberg: Springer-Verlag Berlin Heidelberg, 2006. ISBN: 3-540-26579-1, 978-3-540-26579-5.

[Renn.1986] Renn, Werner: Struktur und Aufbau Prozeßnaher Steuergeräte zur Verkettung in flexiblen Fertigungssystemen. Berlin [u.a.]: Springer, 1986. ISSN 3-540-16150-3, 0-387-16150-3.

[Salv.2001]

[Tuff.1988]

[VDI 3633] Salvendy, Gavriel: Handbook of industrial engineering: Technology and operations management. New York, NY [u.a.] : Wiley, 2001. ISBN: 0-471-33057-4.

Tuffentsammer, Karl; Storr, Alfred; Pritschow, Günter; Warnecke, Hans-Jürgen: Flexibles Fertigungssystem: Beiträge zur Entwicklung des Produktionsprinzips. Weinheim [u.a.] : VCH, 1988. ISBN: 3-527-27706-4.

Simulation von Logistik-, Materialfluß- und Produktionssystemen. Düsseldorf: VDI-Verl., 1993 